\title{
SOLVABILITY OF THE INITIAL VALUE PROBLEM TO A MODEL SYSTEM FOR WATER WAVES
}

\author{
Yuuta Murakami and Tatsuo Iguchi
}

Dedicated to the late Professor Seiji Ukai

\begin{abstract}
We consider the initial value problem to a model system for water waves. The model system is the Euler-Lagrange equations for an approximate Lagrangian which is derived from Luke's Lagrangian for water waves by approximating the velocity potential in the Lagrangian. The model are nonlinear dispersive equations and the hypersurface $t=0$ is characteristic for the model equations. Therefore, the initial data have to be restricted in an infinite dimensional manifold in order to the existence of the solution. Under this necessary condition and a sign condition, which corresponds to a generalized Rayleigh-Taylor sign condition for water waves, on the initial data, we show that the initial value problem is solvable locally in time in Sobolev spaces.
\end{abstract}

\section{Introduction}

In this paper we consider the initial value problem to a model system for water waves. The water wave problem is mathematically formulated as a free boundary problem for an irrotational flow of an inviscid and incompressible fluid under the gravitational field. The basic equations for water waves are complicated due to the nonlinearity of the equations together with the presence of an unknown free surface. Therefore, until now many approximate equations have been proposed and analyzed to understand natural phenomena for water waves. Famous examples of such approximate equations are the shallow water equations $[1,8]$, the Green-Naghdi equations [10, 11], Boussinesq type equations [5], the Korteweg-de Vries equation [18], the Kadomtsev-Petviashvili equation [14], the Benjamin-Bona-Mahony equation [22, 4], the Camassa-Holm equation [9, 6], the Benjamin-Ono equation [3, 21], and so on. All of them are derived from the water wave problem under a shallowness assumption of the water waves, which means that the mean depth of the water is sufficiently small compared to the typical wavelength of the water surface.

2000 Mathematics Subject Classification. 35Q35, 76B15.

Key words and phrases. water waves, shallow water, Luke's Lagrangian.

Received February 25, 2014; revised December 8, 2014. 
On the other hand, in coastal engineering some model equations were derived without using any shallowness assumption of the water waves. It is wellknown that the water wave problem has a variational structure. In fact, J. C. Luke [20] gave a Lagrangian in terms of the velocity potential and the surface variation, and showed that the corresponding Euler-Lagrange equations are the basic equations for water waves. M. Isobe [12] derived model equations for water waves without any shallowness assumption, and then T. Kakinuma [15] extended the model equations for a multi-phase problem for water waves. See also M. Isobe [13] and T. Kakinuma [16, 17]. The model equations are the Euler-Lagrange equations to an approximate Lagrangian, which is obtained by approximating the velocity potential in Luke's Lagrangian. One of the models has the form

$$
\left\{\begin{aligned}
\eta_{t}+ & \nabla \cdot\left(H \nabla \phi^{0}+\frac{1}{3} H^{3} \nabla \phi^{1}-H^{2} \phi^{1} \nabla b\right)=0 \\
H^{2} \eta_{t} & +\nabla \cdot\left(\frac{1}{3} H^{3} \nabla \phi^{0}+\frac{1}{5} H^{5} \nabla \phi^{1}-\frac{1}{2} H^{4} \phi^{1} \nabla b\right) \\
& +H^{2} \nabla b \cdot \nabla \phi^{0}+\frac{1}{2} H^{4} \nabla b \cdot \nabla \phi^{1}-\frac{4}{3} H^{3}\left(1+|\nabla b|^{2}\right) \phi^{1}=0 \\
\phi_{t}^{0}+ & H^{2} \phi_{t}^{1}+g \eta+\frac{1}{2}\left|\nabla \phi^{0}\right|^{2}+\frac{1}{2} H^{4}\left|\nabla \phi^{1}\right|^{2} \\
& +H^{2} \nabla \phi^{0} \cdot \nabla \phi^{1}-2 H \phi^{1} \nabla b \cdot \nabla \phi^{0}-2 H^{3} \phi^{1} \nabla b \cdot \nabla \phi^{1} \\
& +2 H^{2}\left(1+|\nabla b|^{2}\right)\left(\phi^{1}\right)^{2}=0
\end{aligned}\right.
$$

where $\eta=\eta(x, t)$ is the surface elevation, $b=b(x)$ represents the bottom topography, $\phi^{0}=\phi^{0}(x, t)$ and $\phi^{1}=\phi^{1}(x, t)$ are related to the velocity potential $\Phi=\Phi(x, z, t)$ of the water by the approximate formula

$$
\Phi(x, z, t)=\phi^{0}(x, t)+(z-b(x))^{2} \phi^{1}(x, t)
$$

and $H=H(x, t)$ is the depth of water and is given by

$$
H(x, t)=h+\eta(x, t)-b(x) .
$$

Here, $g$ is the gravitational constant and $h$ is the mean depth of the water. Both of them are assumed to be given positive constants. $t$ is the time, $x=$ $\left(x_{1}, x_{2}, \ldots, x_{n}\right) \in \mathbf{R}^{n}$ is the horizontal spatial coordinates, and $z$ is the vertical spatial coordinate. In this paper we will show a unique solvability of the initial value problem for the model system (1.1) under the initial conditions

$$
\left(\eta, \phi^{0}, \phi^{1}\right)=\left(\eta_{0}, \phi_{0}^{0}, \phi_{0}^{1}\right) \quad \text { at } t=0 .
$$

We will explain fundamental properties of the model system (1.1). First, $\left(\eta, \phi^{0}, \phi^{1}\right)=\mathbf{0}$ is the solution of (1.1), which corresponds to the still water with flat water surface. In the case of the flat bottom $b(x) \equiv 0$, the linearized equations of the model system (1.1) around this trivial solution have the form 


$$
\left\{\begin{array}{l}
\eta_{t}+h \Delta \phi^{0}+\frac{h^{3}}{3} \Delta \phi^{1}=0 \\
\eta_{t}+\frac{h}{3} \Delta \phi^{0}+\frac{h^{3}}{5} \Delta \phi^{1}-\frac{4}{3} h \phi^{1}=0 \\
\phi_{t}^{0}+h^{2} \phi_{t}^{1}+g \eta=0 .
\end{array}\right.
$$

This system has a non-trivial solution of the form $\eta(x, t)=\eta_{0} \mathrm{e}^{\mathrm{i}(\xi \cdot x-\omega t)}$ if and only if the wave vector $\xi \in \mathbf{R}^{n}$ and the angular frequency $\omega \in \mathbf{C}$ satisfy the relation

$$
\left(6 h^{2}|\xi|^{2}+15\right) \omega^{2}-g h|\xi|^{2}\left(h^{2}|\xi|^{2}+15\right)=0
$$

which is the linear dispersion relation of the model system (1.1). The phase speed of the wave is given by

$$
\frac{\omega}{|\xi|}= \pm \sqrt{g h} \sqrt{\frac{h^{2}|\xi|^{2}+15}{6 h^{2}|\xi|^{2}+15}} .
$$

Therefore, the model system (1.1) is classified into the dispersive equations. In Section 2 we will compare this dispersion relation to those of well-known model equations. Then, the model system (1.1) turns out to be precise approximate equations for water waves in the shallow water regime, at least, in the linear level.

Secondly, the model system (1.1) is written in the matrix form as

$$
\left(\begin{array}{ccc}
1 & 0 & 0 \\
H^{2} & 0 & 0 \\
0 & 1 & H^{2}
\end{array}\right) \frac{\partial}{\partial t}\left(\begin{array}{c}
\eta \\
\phi^{0} \\
\phi^{1}
\end{array}\right)+\{\text { spatial derivatives }\}=\mathbf{0} .
$$

Since the coefficient matrix always has the zero eigenvalue, the hypersurface $t=0$ in the space-time $\mathbf{R}^{n} \times \mathbf{R}$ is characteristic for the model system (1.1), so that the initial value problem (1.1) and (1.3) is not solvable in general. In fact, if the problem has a solution $\left(\eta, \phi^{0}, \phi^{1}\right)$, then by eliminating the time derivative $\eta_{t}$ from the first two equations in (1.1) we see that the solution has to satisfy the relation

$$
\begin{aligned}
H^{2} \nabla & \cdot\left(H \nabla \phi^{0}+\frac{1}{3} H^{3} \nabla \phi^{1}-H^{2} \phi^{1} \nabla b\right) \\
= & \nabla \cdot\left(\frac{1}{3} H^{3} \nabla \phi^{0}+\frac{1}{5} H^{5} \nabla \phi^{1}-\frac{1}{2} H^{4} \phi^{1} \nabla b\right) \\
& +H^{2} \nabla b \cdot \nabla \phi^{0}+\frac{1}{2} H^{4} \nabla b \cdot \nabla \phi^{1}-\frac{4}{3} H^{3}\left(1+|\nabla b|^{2}\right) \phi^{1} .
\end{aligned}
$$

Therefore, as a necessary condition the initial date $\left(\eta_{0}, \phi_{0}^{0}, \phi_{0}^{1}\right)$ and the bottom topography $b$ have to satisfy the relation (1.6) for the existence of the solution.

Thirdly, the corresponding total energy of the motion for the model system (1.1) is given by 


$$
\begin{gathered}
E(t):=\frac{1}{2} \int_{\mathbf{R}^{n}}\left\{\int_{b(x)}^{h+\eta(x, t)}\left|\nabla_{X}\left(\phi^{0}(x, t)+(z-b(x))^{2} \phi^{1}(x, t)\right)\right|^{2} \mathrm{~d} z\right. \\
\left.+g(\eta(x, t))^{2}\right\} \mathrm{d} x,
\end{gathered}
$$

where $\nabla_{X}=\left(\nabla, \partial_{z}\right)$. In fact, we can see that the energy function $E(t)$ is a conserved quantity in time for any smooth solution $\left(\eta, \phi^{0}, \phi^{1}\right)$ of the model system (1.1) (see Lemma 5.1). Under a physically reasonable condition on the water surface $\eta$ and the bottom $b$ we have an equivalence

$$
E(t) \simeq \int_{\mathbf{R}^{n}}\left\{\left|\nabla \phi^{0}(x, t)\right|^{2}+\left|\nabla \phi^{1}(x, t)\right|^{2}+\left(\phi^{1}(x, t)\right)^{2}+(\eta(x, t))^{2}\right\} \mathrm{d} x .
$$

Therefore, it is natural to work in the class $\eta, \nabla \phi^{0} \in C\left([0, T] ; H^{m}\right)$ and $\phi^{1} \in C\left([0, T] ; H^{m+1}\right)$, where $H^{m}=W^{m, 2}\left(\mathbf{R}^{n}\right)$ is the standard $L^{2}$ Sobolev space of order $m$ on $\mathbf{R}^{n}$. We note that the water wave problem has a conserved energy

$$
E_{W W}(t):=\frac{\rho}{2} \int_{\mathbf{R}^{n}}\left\{\int_{b(x)}^{h+\eta(x, t)}\left|\nabla_{X} \Phi(x, z, t)\right|^{2} \mathrm{~d} z+g(\eta(x, t))^{2}\right\} \mathrm{d} x
$$

where $\rho$ is the constant density of the water. This is a sum of the kinetic and the potential energies. The energy function (1.7) is obtained by this energy function $E_{W W}$ with the velocity potential $\Phi$ replaced by the approximate one (1.2).

Finally, it is well known that the well-posedness of the initial value problem for the full water wave problem may be broken unless a generalized RayleighTaylor sign condition $-\frac{\partial p}{\partial N} \geq c_{0}>0$ on the water surface is satisfied, where $p$ is the pressure and $N$ is the unit outward normal on the water surface (see, for example, [2]). S. Wu $[23,24]$ showed that this condition always holds for any smooth non-self-intersecting surface in the case of infinite depth. In the case with variable bottom, D. Lannes [19] gave a relation between this condition and the bottom topography. However, as far as the authors know, no example of the bottom topography is given for which the sign condition does not hold. We note that the sign condition is equivalent to $-\partial_{z} p \geq c_{0}>0$ because the pressure $p$ is constant on the water surface. By using Bernoulli's law

$$
\Phi_{t}+\frac{1}{2}\left|\nabla_{X} \Phi\right|^{2}+\frac{1}{\rho}\left(p-p_{0}\right)+g(z-h) \equiv 0
$$

and the approximate formula (1.2), the sign condition can be written in term of our unknowns $\left(\eta, \phi^{0}, \phi^{1}\right)$ and $b$ as $a(x, t) \geq c_{0}>0$, where

$$
\begin{aligned}
a:= & g+2 H \phi_{t}^{1}+2 H^{3}\left|\nabla \phi^{1}\right|^{2}+2 H \nabla \phi^{0} \cdot \nabla \phi^{1}-2 \phi^{1} \nabla b \cdot \nabla \phi^{0} \\
& -6 H^{2} \phi^{1} \nabla b \cdot \nabla \phi^{1}+4 H\left(1+|\nabla b|^{2}\right)\left(\phi^{1}\right)^{2} .
\end{aligned}
$$


In fact, we have $-\frac{1}{\rho} \partial_{z} p=g+\partial_{z} \Phi_{t}+\nabla_{X} \partial_{z} \Phi \cdot \nabla_{X} \Phi=a$ on $z=h+\eta(x, t)$. In this paper, we assume that this sign condition is satisfied at the initial time $t=0$.

Now, we are ready to give our main result in this paper.

THEOREM 1.1. Let $g, h, c_{0}, M_{0}$ be positive constants and $m$ an integer such that $m>\frac{n}{2}+1$. There exists a time $T>0$ such that if the initial data $\left(\eta_{0}, \phi_{0}^{0}, \phi_{0}^{1}\right)$ and $b$ satisfy the relation (1.6) and

$$
\left\{\begin{array}{l}
\left\|\eta_{0}\right\|_{m}+\left\|\nabla \phi_{0}^{0}\right\|_{m}+\left\|\phi_{0}^{1}\right\|_{m+1}+\|b\|_{W^{m+2, \infty}} \leq M_{0}, \\
h+\eta_{0}(x)-b(x) \geq c_{0}, \quad a(x, 0) \geq c_{0} \quad \text { for } x \in \mathbf{R}^{n},
\end{array}\right.
$$

then the initial value problem (1.1) and (1.3) has a unique solution $\left(\eta, \phi^{0}, \phi^{1}\right)$ satisfying

$$
\eta, \nabla \phi^{0} \in C\left([0, T] ; H^{m}\right), \quad \phi^{1} \in C\left([0, T] ; H^{m+1}\right) .
$$

Remark 1.1. (1) If we impose an additional condition $\phi_{0}^{0} \in H^{m+1}$, then the solution satisfies an additional regularity $\phi^{0} \in C\left([0, T] ; H^{m+1}\right)$.

(2) In the sign condition $a(x, 0) \geq c_{0}>0$ we have the quantity $\phi_{t}^{1}(x, 0)$ which should be written in terms of the initial data. Although the hypersurface $t=0$ is characteristic for the system $(1.1)$, we can express $\phi_{t}^{1}(x, 0)$ in terms of the initial data and $b$. For details, we refer to Remark 3.1 in Section 3.

The contents of this paper are as follows. In Section 2 we sketch the derivation of the model system (1.1) and discuss an accuracy of the approximation. In Section 3 we transform the model system (1.1) to a system of equations for which the hypersurface $t=0$ is noncharacteristic by using the necessary condition (1.6). In Section 4 we construct the solution of the initial value problem to the transformed equations by using a standard parabolic regularization. In Section 5 we show that the solution constructed in Section 4 is the solution of the model system (1.1) if the initial data satisfy the necessary condition (1.6).

Notation. We denote by $W^{m, p}\left(\mathbf{R}^{n}\right)$ the $L^{p}$ Sobolev space of order $m$ on $\mathbf{R}^{n}$. The norms of the Lebesgue space $L^{p}\left(\mathbf{R}^{n}\right)$ and the Sobolev space $H^{m}=W^{m, 2}\left(\mathbf{R}^{n}\right)$ are denoted by $|\cdot|_{p}$ and $\|\cdot\|_{m}$, respectively. The $L^{2}$ norm and inner product are simply denoted by $\|\cdot\|$ and $(\cdot, \cdot)$, respectively. $\mathscr{P}(X ; Y)$ is the set of all polynomials in $X=\left(X_{1}, \ldots, X_{l_{1}}\right)$ and $Y=\left(Y_{1}, \ldots, Y_{l_{2}}\right)$ which are homogeneous of degree 2 in $Y . \quad D^{m} u=\left\{\left(\frac{\partial}{\partial x}\right)^{\alpha} u|| \alpha \mid=m\right\}$ is the set of all partial derivatives of $u$ of order $m$ in $x$. $[P, Q]=P Q-Q P$ denotes the commutator. 


\section{The model system}

Here, we will sketch the derivation of the model system (1.1) for water waves. As mentioned in the introduction L. C. Luke [20] showed that the water wave problem has a variational structure by giving a Lagrangian in terms of $\Phi$ and $\eta$. His Lagrangian has the form

$$
\mathscr{L}(\Phi, \eta)=\int_{b(x)}^{h+\eta(x, t)}\left(\Phi_{t}(x, z, t)+\frac{1}{2}\left|\nabla_{X} \Phi(x, z, t)\right|^{2}+g(z-h)\right) \mathrm{d} z
$$

and the action function is

$$
\mathscr{J}(\Phi, \eta)=\int_{t_{0}}^{t_{1}} \int_{\Omega} \mathscr{L}(\Phi, \eta) \mathrm{d} x \mathrm{~d} t
$$

where $\Omega$ is an appropriate region in $\mathbf{R}^{n}$. In view of Bernoulli's law (1.8) this Lagrangian is essentially the integral of the pressure $p$ in the vertical direction of the water region. L. C. Luke showed that the corresponding Euler-Lagrange equation is exactly the basic equations for water waves. M. Isobe [12] and T. Kakinuma [15] approximated the velocity potential in Luke's Lagrangian as

$$
\Phi(x, z, t) \simeq \sum_{k=0}^{K} \Psi_{k}(z ; b) \phi^{k}(x, t),
$$

where $\left\{\Psi_{k}\right\}$ is an appropriate function system, and derived an approximate Lagrangian for $\left(\eta, \phi^{0}, \phi^{1}, \ldots, \phi^{K}\right)$. Their model equations are the corresponding Euler-Lagrange equations. If we approximate $\Phi$ by $\phi^{0}$ a function independent of the vertical spatial variable $z$, then Luke's Lagrangian is approximate by

$$
\mathscr{L}_{0}\left(\phi^{0}, \eta\right)=\left(\phi_{t}^{0}+\frac{1}{2}\left|\nabla \phi^{0}\right|^{2}\right)(h+\eta-b)+\frac{1}{2} g\left(\eta^{2}-(b-h)^{2}\right) .
$$

The corresponding Euler-Lagrange equations are the shallow water equations

$$
\left\{\begin{array}{l}
\eta_{t}+\nabla \cdot\left((h+\eta-b) \nabla \phi^{0}\right)=0 \\
\phi_{t}^{0}+\frac{1}{2}\left|\nabla \phi^{0}\right|^{2}+g \eta=0 .
\end{array}\right.
$$

If we approximate $\Phi$ by the formula (1.2) in Luke's Lagrangian, then the corresponding Euler-Lagrange equations are the model system (1.1). In fact, by the variations with respect to $\phi^{0}, \phi^{1}$, and $\eta$, we obtain the first, the second, and the third equations in (1.1), respectively.

Remark 2.1. (1) If we adopt the approximation of the velocity potential

$$
\Phi(x, z, t)=\phi^{0}(x, t)+(z-b(x))^{p} \phi^{1}(x, t)
$$


with a constant $p>0$ in place of the approximation (1.2), then we have slightly different equations than (1.1). However, basic properties of the equations are the same as those of (1.2) and similar existence theorem as Theorem 1.1 holds.

(2) D. Clamond and D. Dutykh [7] derived several model equations for water waves. The models are also Euler-Lagrange equations for approximate Lagrangians which are derived from Luke's Lagrangian for water waves by adding as many variables as possible in order to include the effects of the compressibility of the fluid and of the vorticity of the flow. Therefore, the models are essentially different from the model (1.1).

Next, we will compare the dispersion relation (1.5) to those of well-known model equations. The linear dispersion relations of the shallow water (SW) equations, the Korteweg-de Vries (KdV) equation, the Benjamin-Bona-Mahony $(\mathrm{BBM})$ equation, the Green-Naghdi $(\mathrm{GN})$ equations, and the full water wave (WW) equations are given by

$$
\begin{aligned}
& (\mathrm{SW}) \quad \omega^{2}-g h|\xi|^{2}=0 \\
& (\mathrm{KdV}) \quad \pm \omega-\sqrt{g h} \xi+\frac{1}{6} \sqrt{g h} h^{2} \xi^{3}=0 \\
& (\mathrm{BBM}) \quad \pm\left(1+\frac{1}{6} h^{2} \xi^{2}\right) \omega-\sqrt{g h} \xi=0 \\
& (\mathrm{GN}) \quad\left(1+\frac{1}{3} h^{2}|\xi|^{2}\right) \omega^{2}-g h|\xi|^{2}=0 \\
& (\mathrm{WW}) \quad \omega^{2}-g|\xi| \tanh (h|\xi|)=0
\end{aligned}
$$

so that the corresponding phase speeds $c_{S W}, c_{K d V}, c_{B B M}, c_{G N}$, and $c_{W W}$ are given by $c_{S W}= \pm \sqrt{g h}, c_{K d V}(\xi)= \pm \sqrt{g h}\left(1-\frac{1}{6} h^{2} \xi^{2}\right), c_{B B M}(\xi)= \pm \frac{\sqrt{g h}}{1+\frac{1}{6} h^{2} \xi^{2}}, c_{G N}(\xi)=$ $\pm \sqrt{\frac{g h}{1+\frac{1}{3} h^{2}|\xi|^{2}}}$, and $c_{W W}(\xi)= \pm \sqrt{\frac{g \tanh (h|\xi|)}{|\xi|}}$, respectively. On the other hand, the phase speed $c_{I K}$ of the model due to Isobe and Kakinuma (IK) is given by

$$
c_{I K}(\xi)= \pm \sqrt{g h \frac{1+\frac{1}{15} h^{2}|\xi|^{2}}{1+\frac{2}{5} h^{2}|\xi|^{2}}}
$$

We note that as $\xi \rightarrow 0$ all of them converge to $c_{S W}$ and that a Taylor approximation of $c_{B B M}(\xi), c_{G N}(\xi), c_{I K}(\xi)$, and $c_{W W}(\xi)$ coincides with $c_{K d V}(\xi)$. Moreover, $c_{B B M}(\xi)$ is the $[0 / 2]$ Padé approximant of $c_{W W}(\xi),\left(c_{G N}(\xi)\right)^{2}$ is the $[0 / 2]$ Padé approximant of $\left(c_{W W}(\xi)\right)^{2}$, and $\left(c_{I K}(\xi)\right)^{2}$ is the [2/2] Padé approximant of $\left(c_{W W}(\xi)\right)^{2}$. Therefore, among them $c_{I K}(\xi)$ is the best approximation of $c_{W W}(\xi)$ in the shallow water regime $h|\xi| \ll 1$. In fact, we have the following Taylor expansions 


$$
\begin{aligned}
c_{W W}(\xi) & = \pm \sqrt{g h}\left(1-\frac{1}{6}(h|\xi|)^{2}+\frac{19}{360}(h|\xi|)^{4}-\frac{55}{3024}(h|\xi|)^{6}\right)+O\left((h|\xi|)^{8}\right) \\
c_{I K}(\xi) & = \pm \sqrt{g h}\left(1-\frac{1}{6}(h|\xi|)^{2}+\frac{19}{360}(h|\xi|)^{4}-\frac{193}{10800}(h|\xi|)^{6}\right)+O\left((h|\xi|)^{8}\right)
\end{aligned}
$$

whereas

$$
c_{G N}(\xi)= \pm \sqrt{g h}\left(1-\frac{1}{6}(h|\xi|)^{2}+\frac{1}{24}(h|\xi|)^{4}\right)+O\left((h|\xi|)^{6}\right) .
$$

Once we admit higher order derivative terms in the approximate equations, we may obtain good model equations whose dispersion relation is as precise as the model (1.1). However, higher order derivative terms are troublesome in a numerical computation. The model (1.1) contains only up to second order derivative terms. This is an advantage of the model.

Finally, we will give one more remark on the consistency of the linearized equations (1.4) with the full water wave equations in the shallow water approximation. As was shown by J. Boussinesq [5], in the case of the flat bottom the velocity potential $\Phi$ can be expanded in a Taylor series with respect to the vertical spatial variable as

$$
\Phi(x, z, t)=\sum_{n=0}^{\infty} \frac{z^{2 n}}{(2 n) !}(-\Delta)^{n} \phi_{0}(x, t)
$$

where $\phi_{0}$ is the trace of the velocity potential $\Phi$ on the bottom. In view of our approximation (1.2), one might expect that the unknown functions $\phi^{0}$ and $\phi^{1}$ are related with $\phi_{0}$ by $\phi^{0}=\phi_{0}$ and $\phi^{1}=-\frac{1}{2} \Delta \phi_{0}$ and that the relation $\phi^{1}=-\frac{1}{2} \Delta \phi^{0}$ holds. However, it is not true and the solution $\left(\eta, \phi^{0} \phi^{1}\right)$ of (1.4) does not satisfy this relation $\phi^{1}=-\frac{1}{2} \Delta \phi^{0}$ but the relation

$$
\left(1+\frac{1}{10} h^{2} \Delta\right) \phi^{1}=-\frac{1}{2} \Delta \phi^{0}
$$

which comes from the linearized version of the relation (1.6). Therefore, the relation $\phi^{1}=-\frac{1}{2} \Delta \phi^{0}$ is satisfied approximately as $h^{2}{\widehat{\phi^{1}}}^{1}=\frac{1}{2}(h|\xi|)^{2} \widehat{\phi}^{0}+O\left((h|\xi|)^{4}\right)$ in the shallow water regime $h|\xi| \ll 1$, where $\hat{\cdot}$ denotes the Fourier transform in the horizontal spatial variables $x$. Moreover, in view of our approximation (1.2) again, the trace of the velocity potential $\Phi$ on the water surface should be given by

$$
\phi(x, t):=\phi^{0}(x, t)+h^{2} \phi^{1}(x, t)
$$

in terms of our variables $\phi^{0}$ and $\phi^{1}$. It follows from (2.1) and (2.2) that $\phi^{0}$ and $\phi^{1}$ can be expressed in terms of $\phi$ as 


$$
\left\{\begin{array}{l}
\phi^{0}=\left(1-\frac{2}{5} h^{2} \Delta\right)^{-1}\left(1+\frac{1}{10} h^{2} \Delta\right) \phi, \\
\phi^{1}=-\frac{1}{2}\left(1-\frac{2}{5} h^{2} \Delta\right)^{-1} \Delta \phi,
\end{array}\right.
$$

so that the first two equations can be transformed into

$$
\eta_{t}+h\left(1-\frac{2}{5} h^{2} \Delta\right)^{-1}\left(1-\frac{1}{15} h^{2} \Delta\right) \Delta \phi=0 .
$$

Therefore, if $\left(\eta, \phi^{0}, \phi^{1}\right)$ is a solution of (1.4) and if $\phi$ is defined by (2.2), then $(\eta, \phi)$ satisfies

$$
\left\{\begin{array}{l}
\hat{\eta}_{t}-|\xi| \tanh (h|\xi|) \hat{\phi}=O\left(|\xi|(h|\xi|)^{7}\right), \\
\hat{\phi}_{t}+g \hat{\eta}=0
\end{array}\right.
$$

This means that $(\eta, \phi)$ satisfies the linearized water wave equations up to order $|\xi|(h|\xi|)^{7}$. Since the shallow water equations and the Green-Naghdi equations are approximations up to order $|\xi|(h|\xi|)^{3}$ and $|\xi|(h|\xi|)^{5}$, respectively, the model (1.1) due to Isobe and Kakinuma is in fact fit in the shallow water theory, at least, in the linear level.

These considerations motivate us to give a mathematically rigorous justification of the model (1.1). We postpone this subject in the future research.

\section{Transformation of the system}

Our basic idea to solve the initial value problem (1.1) and (1.3) is to transform the model system (1.1) into a system of equations by using the relation (1.6) for which the hypersurface $t=0$ is noncharacteristic and the initial value problem is well-posed in Sobolev spaces.

We introduce second order differential operators $L_{11}=L_{11}(H, b), L_{12}=$ $L_{12}(H, b)$, and $L_{22}=L_{22}(H, b)$ depending on the depth of the water $H$ and the bottom topography $b$ by

$$
\left\{\begin{aligned}
L_{11} \psi^{0}:= & -\nabla \cdot\left(H \nabla \psi^{0}\right) \\
L_{12} \psi^{1}:= & -\nabla \cdot\left(\frac{1}{3} H^{3} \nabla \psi^{1}-H^{2} \psi^{1} \nabla b\right) \\
L_{22} \psi^{1}:= & -\nabla \cdot\left(\frac{1}{5} H^{5} \nabla \psi^{1}-\frac{1}{2} H^{4} \psi^{1} \nabla b\right)-\frac{1}{2} H^{4} \nabla b \cdot \nabla \psi^{1} \\
& +\frac{4}{3} H^{3}\left(1+|\nabla b|^{2}\right) \psi^{1} .
\end{aligned}\right.
$$

Then, we see that $L_{11}$ and $L_{22}$ are symmetric in $L^{2}\left(\mathbf{R}^{n}\right)$ and the adjoint operator $L_{12}^{*}$ of $L_{12}$ is given by

$$
L_{12}^{*} \psi^{0}=-\nabla \cdot\left(\frac{1}{3} H^{3} \nabla \psi^{0}\right)-H^{2} \nabla b \cdot \nabla \psi^{0} .
$$


Therefore, the model system (1.1) and the relation (1.6) can be written as

$$
\left\{\begin{array}{l}
\eta_{t}-L_{11} \phi^{0}-L_{12} \phi^{1}=0 \\
H^{2} \eta_{t}-L_{12}^{*} \phi^{0}-L_{22} \phi^{1}=0 \\
\phi_{t}^{0}+H^{2} \phi_{t}^{1}+F_{1}=0
\end{array}\right.
$$

and

$$
H^{2}\left(L_{11} \phi^{0}+L_{12} \phi^{1}\right)=L_{12}^{*} \phi^{0}+L_{22} \phi^{1}
$$

respectively, where

$$
\begin{aligned}
F_{1}:= & g \eta+\frac{1}{2}\left|\nabla \phi^{0}\right|^{2}+\frac{1}{2} H^{4}\left|\nabla \phi^{1}\right|^{2}+H^{2} \nabla \phi^{0} \cdot \nabla \phi^{1} \\
& -2 H \phi^{1} \nabla b \cdot \nabla \phi^{0}-2 H^{3} \phi^{1} \nabla b \cdot \nabla \phi^{1}+2 H^{2}\left(1+|\nabla b|^{2}\right)\left(\phi^{1}\right)^{2} .
\end{aligned}
$$

Differentiating (3.3) with respect to $t$ and using the first equation in (3.2) to eliminate $\eta_{t}$ we obtain

$$
\left(H^{2} L_{11}-L_{12}^{*}\right) \phi_{t}^{0}-\left(L_{22}-H^{2} L_{12}\right) \phi_{t}^{1}=F_{2},
$$

where

$$
\left\{\begin{aligned}
F_{2}:= & -F_{3} \nabla \cdot\left(H \nabla \phi^{0}+\frac{1}{3} H^{3} \nabla \phi^{1}-H^{2} \phi^{1} \nabla b\right), \\
F_{3}:= & 2 H^{2} \Delta \phi^{0}+\frac{2}{3} H^{4} \Delta \phi^{1}-2 H \nabla b \cdot \nabla \phi^{0} \\
& -4 H^{3} \nabla b \cdot \nabla \phi^{1}-2 H^{3} \phi^{1} \Delta b+4 H^{2}\left(1+|\nabla b|^{2}\right) \phi^{1} .
\end{aligned}\right.
$$

We also introduce second order differential operator $L_{0}=L_{0}(H, b)$ and $L_{1}=$ $L_{1}(H, b)$ by

$$
\begin{gathered}
L_{0} \psi^{0}:=\left(L_{11}-H^{-2} L_{12}^{*}\right) \psi^{0}+\left(H^{-2} L_{22}-L_{12}\right)\left(H^{-2} \psi^{0}\right) \\
=-\frac{8}{15} \nabla \cdot\left(H \nabla \psi^{0}\right)+H^{-1}\left(\frac{4}{3}\left(1+|\nabla b|^{2}\right)+2 \nabla b \cdot \nabla H+\frac{4}{5}|\nabla H|^{2}\right. \\
\left.-\frac{4}{15} H \Delta H-\frac{1}{2} H \Delta b\right) \psi^{0}
\end{gathered}
$$

and

$$
\begin{aligned}
L_{1} \psi^{1}:= & \left(H^{2} L_{11}-L_{12}^{*}\right)\left(H^{2} \psi^{1}\right)+\left(L_{22}-H^{2} L_{12}\right) \psi^{1} \\
= & -\frac{8}{15} \nabla \cdot\left(H^{5} \nabla \psi^{1}\right) \\
& +H^{3}\left(\frac{4}{3}\left(1+|\nabla b|^{2}\right)+2 \nabla b \cdot \nabla H-\frac{4}{3}|\nabla H|^{2}-\frac{4}{3} H \Delta H-\frac{1}{2} H \Delta b\right) \psi^{1}
\end{aligned}
$$


respectively. $\quad L_{0}$ and $L_{1}$ are also symmetric in $L^{2}\left(\mathbf{R}^{n}\right)$ and are related each other by

$$
H^{2} L_{0}\left(H^{2} \psi^{1}\right)=L_{1} \psi^{1}
$$

It follows from the third equation in (3.2) that $\phi_{t}^{1}=-H^{-2}\left(\phi_{t}^{0}+F_{1}\right)$ and $\phi_{t}^{0}=$ $-H^{2} \phi_{t}^{1}-F_{1}$. Plugging these into (3.5) we obtain

$$
\left\{\begin{array}{l}
L_{0} \phi_{t}^{0}+\left(H^{-2} L_{22}-L_{12}\right)\left(H^{-2} F_{1}\right)=H^{-2} F_{2}, \\
L_{1} \phi_{t}^{1}+\left(H^{2} L_{11}-L_{12}^{*}\right) F_{1}=-F_{2} .
\end{array}\right.
$$

These should be evolution equations for unknowns $\phi^{0}$ and $\phi^{1}$.

Remark 3.1. As mentioned in Remark 1.1 (2) we have to express $\phi_{t}^{1}(x, 0)$ in terms of the initial data and $b$. Such an expression is given by

$$
\phi_{t}^{1}(\cdot, 0)=-\left.L_{1}^{-1}\left(F_{2}+\left(H^{2} L_{11}-L_{12}^{*}\right) F_{1}\right)\right|_{t=0} .
$$

The invertibility of the operator $L_{1}$ will be proved later. See Lemmas 3.1 and 3.2.

Now, we are going to determine the principal parts of the left-hand sides of the equations in (3.10). It is easy to see that

$$
\left\{\begin{aligned}
\left(H^{-2} L_{22}-L_{12}\right) \psi & \\
=\frac{2}{15} H^{3} \Delta \psi & -H^{2} \nabla b \cdot \nabla \psi+\left(\frac{4}{3} H\left(1+|\nabla b|^{2}\right)-\frac{1}{2} H^{2} \Delta b\right) \psi \\
\left(H^{2} L_{11}-L_{12}^{*}\right) \psi & =-\frac{2}{3} H^{3} \Delta \psi+H^{2} \nabla b \cdot \nabla \psi
\end{aligned}\right.
$$

and that

$$
\Delta F_{1}=a_{1} \Delta \eta+\boldsymbol{u} \cdot \nabla \Delta \phi^{0}+H^{2} \boldsymbol{u} \cdot \nabla \Delta \phi^{1}+F_{4}
$$

where

$$
\left\{\begin{array}{c}
a_{1}:=g+2 H^{3}\left|\nabla \phi^{1}\right|^{2}+2 H \nabla \phi^{0} \cdot \nabla \phi^{1}-2 \phi^{1} \nabla b \cdot \nabla \phi^{0} \\
\quad-6 H^{2} \phi^{1} \nabla b \cdot \nabla \phi^{1}+4 H\left(1+|\nabla b|^{2}\right)\left(\phi^{1}\right)^{2} \\
\boldsymbol{u}:=\nabla \phi^{0}+H^{2} \nabla \phi^{1}-2 H \phi^{1} \nabla b
\end{array}\right.
$$

and $F_{4}$ is a collection of lower order terms, more precisely,

$$
F_{4} \in \mathscr{P}\left(H, D b, D^{2} b, D^{3} b, D \eta ; \phi^{1}, D \phi, D^{2} \phi\right),
$$

where $\phi=\left(\phi^{0}, \phi^{1}\right)$. Here, we note that $\boldsymbol{u}$ is the horizontal velocity on the water surface. In fact, in view of $(1.2)$ we have $(\nabla \Phi)(x, h+\eta(x, t), t)=\boldsymbol{u}(x, t)$. On the other hand, it follows from the relation (1.6) that 


$$
\begin{aligned}
\frac{2}{3} H \Delta \phi^{0}+\frac{2}{15} H^{3} \Delta \phi^{1}= & \nabla b \cdot \nabla \phi^{0}+H^{2} \nabla b \cdot \nabla \phi^{1} \\
& +\left(\frac{1}{2} H^{2} \Delta b-\frac{4}{3} H\left(1+|\nabla b|^{2}\right)\right) \phi^{1},
\end{aligned}
$$

which expresses $\Delta \phi^{0}$ in terms of $\Delta \phi^{1}$ with lower order terms and vice versa. By (3.12), (3.13), and (3.16), we can rewrite equations in (3.10) as

$$
\left\{\begin{array}{l}
P_{0} \phi_{t}^{0}-4 \nabla \cdot\left(H(\boldsymbol{u} \cdot \nabla) \nabla \phi^{0}\right)+\nabla \cdot\left(\left(H a_{1}-2 F_{1}\right) \nabla \eta\right)=G_{0} \\
P_{1} \phi_{t}^{1}-\frac{4}{5} \nabla \cdot\left(H^{5}(\boldsymbol{u} \cdot \nabla) \nabla \phi^{1}\right)-\nabla \cdot\left(H^{3} a_{1} \nabla \eta\right)=G_{1}
\end{array}\right.
$$

where

$$
P_{0}=P_{0}(H, b):=\frac{15}{2} L_{0}(H, b), \quad P_{1}=P_{1}(H, b):=\frac{3}{2} L_{1}(H, b),
$$

and $G_{0}$ and $G_{1}$ are collections of lower order terms, more precisely,

$$
G_{0}, G_{1} \in \mathscr{P}\left(H, H^{-1}, D b, D^{2} b, D^{3} b, D \eta ; \phi^{1}, D \phi, D^{2} \phi\right) .
$$

Next, we will derive appropriate evolution equation for $\eta$, which together with the above evolution equations for $\phi=\left(\phi^{0}, \phi^{1}\right)$ should have a good symmetry. The first equation in (1.1) can be written as

$$
\eta_{t}+\boldsymbol{u} \cdot \nabla H+H \Delta \phi^{0}+\frac{1}{3} H^{3} \Delta \phi^{1}-H^{2} \nabla \cdot\left(\phi^{1} \nabla b\right)=0,
$$

which together with (3.16) implies that

$$
9 \eta_{t}+9 \boldsymbol{u} \cdot \nabla \eta-H \Delta \phi^{0}+H^{3} \Delta \phi^{1}=G_{2},
$$

where $G_{2}$ is a collection of lower order terms, more precisely,

$$
\begin{aligned}
G_{2}:= & 9 H^{2} \nabla \cdot\left(\phi^{1} \nabla b\right) \\
& -15\left\{\nabla b \cdot \nabla \phi^{0}+H^{2} \nabla b \cdot \nabla \phi^{1}+\left(\frac{1}{2} H^{2} \Delta b-\frac{4}{3} H\left(1+|\nabla b|^{2}\right)\right) \phi^{1}\right\} .
\end{aligned}
$$

Now, we have derived reduced equations

$$
\left\{\begin{array}{l}
P_{0} \phi_{t}^{0}-4 \nabla \cdot\left(H(\boldsymbol{u} \cdot \nabla) \nabla \phi^{0}\right)+\nabla \cdot\left(\left(H a_{1}-2 F_{1}\right) \nabla \eta\right)=G_{0} \\
P_{1} \phi_{t}^{1}-\frac{4}{5} \nabla \cdot\left(H^{5}(\boldsymbol{u} \cdot \nabla) \nabla \phi^{1}\right)-\nabla \cdot\left(H^{3} a_{1} \nabla \eta\right)=G_{1} \\
9 \eta_{t}+9 \boldsymbol{u} \cdot \nabla \eta-H \Delta \phi^{0}+H^{3} \Delta \phi^{1}=G_{2}
\end{array}\right.
$$

for which the following initial conditions are imposed.

$$
\left(\eta, \phi^{0}, \phi^{1}\right)=\left(\eta_{0}, \phi_{0}^{0}, \phi_{0}^{1}\right) \quad \text { at } t=0 .
$$


The hypersurface $t=0$ is noncharacteristic for the reduced system (3.21). In fact, the second order differential operators $P_{0}$ and $P_{1}$ are positive in $L^{2}\left(\mathbf{R}^{n}\right)$ as shown in the following lemma.

Lemma 3.1. Suppose that $0<c_{0} \leq H(x) \leq c_{1}$ and $\nabla b \in L^{\infty}\left(\mathbf{R}^{n}\right)$. There exists a positive constant $C=C\left(c_{0}, c_{1}\right)$ depending only on $c_{0}$ and $c_{1}$ such that we have

$$
\left(P_{0} \psi^{0}, \psi^{0}\right) \geq C^{-1}\left\|\psi^{0}\right\|_{1}^{2}, \quad\left(P_{1} \psi^{1}, \psi^{1}\right) \geq C^{-1}\left\|\psi^{1}\right\|_{1}^{2} .
$$

Proof. In view of (3.17) it is sufficient to show the estimates for the operators $L_{0}$ and $L_{1}$. By direct calculation we have

$$
\begin{aligned}
I:= & \int_{\mathbf{R}^{n}}\left\{\int_{b(x)}^{h+\eta(x)}\left|\nabla_{X}\left(\psi^{0}(x, t)+(z-b(x))^{2} \psi^{1}(x, t)\right)\right|^{2} \mathrm{~d} z\right\} \mathrm{d} x \\
= & \int_{\mathbf{R}^{n}}\left\{H\left|\nabla \psi^{0}\right|^{2}+\frac{2}{3} H^{3} \nabla \psi^{0} \cdot \nabla \psi^{1}+\frac{1}{5} H^{5}\left|\nabla \psi^{1}\right|^{2}-2 H^{2} \psi^{1} \nabla b \cdot \nabla \psi^{0}\right. \\
& \left.\quad-H^{4} \psi^{1} \nabla b \cdot \nabla \psi^{1}+\frac{4}{3} H^{3}\left(1+|\nabla b|^{2}\right)\left(\psi^{1}\right)^{2}\right\} \mathrm{d} x \\
= & \left(L_{11} \psi^{0}+L_{12} \psi^{1}, \psi^{0}\right)+\left(L_{12}^{*} \psi^{0}+L_{22} \psi^{1}, \psi^{1}\right) .
\end{aligned}
$$

On the other hand, we rewrite this integral as

$$
\begin{gathered}
I=\int_{\mathbf{R}^{n}}\left\{\int _ { 0 } ^ { H ( x ) } \left\{\left|\nabla \psi^{0}(x)-2 z \psi^{1}(x) \nabla b(x)+z^{2} \nabla \psi^{1}(x)\right|^{2}\right.\right. \\
\left.\left.\quad+4 z^{2}\left(\psi^{1}(x)\right)^{2}\right\} \mathrm{~d} z\right\} \mathrm{d} x \\
\simeq \int_{\mathbf{R}^{n}}\left\{H\left|\nabla \psi^{0}\right|^{2}+H^{3}\left(1+|\nabla b|^{2}\right)\left(\psi^{1}\right)^{2}+H^{5}\left|\nabla \psi^{1}\right|^{2}\right\} \mathrm{d} x,
\end{gathered}
$$

where $\simeq$ represents an equivalence of these integrals. Therefore, there exists a positive constant $C=C\left(c_{0}\right)$ such that

$$
\left(L_{11} \psi^{0}+L_{12} \psi^{1}, \psi^{0}\right)+\left(L_{12}^{*} \psi^{0}+L_{22} \psi^{1}, \psi^{1}\right) \geq C^{-1}\left(\left\|\nabla \psi^{0}\right\|^{2}+\left\|\psi^{1}\right\|^{2}+\left\|\nabla \psi^{1}\right\|^{2}\right) .
$$

By the definitions (3.7) and (3.8) of the operators $L_{0}$ and $L_{1}$ we see that

$$
\begin{aligned}
\left(L_{0} \psi^{0}, \psi^{0}\right) & =\left(L_{11} \psi^{0}+L_{12}\left(-H^{-2} \psi^{0}\right), \psi^{0}\right)+\left(L_{12}^{*} \psi^{0}+L_{22}\left(-H^{-2} \psi^{0}\right),\left(-H^{-2} \psi^{0}\right)\right) \\
& \geq C^{-1}\left(\left\|\nabla \psi^{0}\right\|^{2}+\left\|H^{-2} \psi^{0}\right\|^{2}+\left\|\nabla\left(H^{-2} \psi^{0}\right)\right\|^{2}\right) \\
& \geq C^{-1}\left(\left\|\nabla \psi^{0}\right\|^{2}+c_{1}^{-4}\left\|\psi^{0}\right\|^{2}\right)
\end{aligned}
$$

and that 


$$
\begin{aligned}
\left(L_{1} \psi^{1}, \psi^{1}\right) & =\left(L_{11}\left(-H^{2} \psi^{1}\right)+L_{12} \psi^{1},\left(-H^{2} \psi^{1}\right)\right)+\left(L_{12}^{*}\left(-H^{2} \psi^{1}\right)+L_{22} \psi^{1}, \psi^{1}\right) \\
& \geq C^{-1}\left(\left\|\nabla\left(H^{2} \nabla \psi^{1}\right)\right\|^{2}+\left\|\psi^{1}\right\|^{2}+\left\|\nabla \psi^{1}\right\|^{2}\right) \\
& \geq C^{-1}\left(\left\|\psi^{1}\right\|^{2}+\left\|\nabla \psi^{1}\right\|^{2}\right),
\end{aligned}
$$

which give the desired estimates.

By this lemma, the explicit expressions (3.7) and (3.8) of the operators $L_{0}$ and $L_{1}$, the standard elliptic estimates, and the definition (3.17) of the operator $P_{0}$ and $P_{1}$, we can easily obtain the following lemma.

Lemma 3.2. Let $h, c_{0}, M$ be positive constants and $m$ an integer such that $m>\frac{n}{2}+1$. There exists a positive constant $C=C\left(h, c_{0}, M\right)$ such that if $\eta$ and $b$ satisfy

$$
\left\{\begin{array}{l}
\|\eta\|_{m}+\|b\|_{W^{m+1, \infty}\left(\mathbf{R}^{n}\right)} \leq M \\
c_{0} \leq H(x)=h+\eta(x)-b(x) \quad \text { for } x \in \mathbf{R}^{n}
\end{array}\right.
$$

then for $1 \leq k \leq m$ we have

$$
\left\|P_{0}^{-1} f\right\|_{k}+\left\|P_{1}^{-1} f\right\|_{k} \leq C\|f\|_{k-2}
$$

\section{Construction of the solution to the reduced system}

In this section we will construct the solution to the initial value problem for the reduced system (3.21) and (3.22) derived in the previous section by a standard parabolic regularization of the equations, that is,

$$
\left\{\begin{array}{l}
P_{0}\left(\phi_{t}^{0}-\varepsilon \Delta \phi^{0}\right)-4 \nabla \cdot\left(H(\boldsymbol{u} \cdot \nabla) \nabla \phi^{0}\right)+\nabla \cdot\left(\left(H a_{1}-2 F_{1}\right) \nabla \eta\right)=G_{0}, \\
P_{1}\left(\phi_{t}^{1}-\varepsilon \Delta \phi^{1}\right)-\frac{4}{5} \nabla \cdot\left(H^{5}(\boldsymbol{u} \cdot \nabla) \nabla \phi^{1}\right)-\nabla \cdot\left(H^{3} a_{1} \nabla \eta\right)=G_{1}, \\
9\left(\eta_{t}-\varepsilon \Delta \eta\right)+9 \boldsymbol{u} \cdot \nabla \eta-H \Delta \phi^{0}+H^{3} \Delta \phi^{1}=G_{2},
\end{array}\right.
$$

where $\varepsilon>0$ is a small regularized parameter. We impose the same initial conditions.

$$
\left(\eta, \phi^{0}, \phi^{1}\right)=\left(\eta_{0}, \phi_{0}^{0}, \phi_{0}^{1}\right) \quad \text { at } t=0 .
$$

We rewrite the regularized system (4.1) as

$$
\left\{\begin{array}{l}
\phi_{t}^{0}-\varepsilon \Delta \phi^{0}=P_{0}^{-1}\left\{G_{0}+4 \nabla \cdot\left(H(\boldsymbol{u} \cdot \nabla) \nabla \phi^{0}\right)-\nabla \cdot\left(\left(H a_{1}-2 F_{1}\right) \nabla \eta\right)\right\}, \\
\phi_{t}^{1}-\varepsilon \Delta \phi^{1}=P_{1}^{-1}\left\{G_{1}+\frac{4}{5} \nabla \cdot\left(H^{5}(\boldsymbol{u} \cdot \nabla) \nabla \phi^{1}\right)+\nabla \cdot\left(H^{3} a_{1} \nabla \eta\right)\right\}, \\
\eta_{t}-\varepsilon \Delta \eta=\frac{1}{9}\left\{G_{2}-9 \boldsymbol{u} \cdot \nabla \eta+H \Delta \phi^{0}-H^{3} \Delta \phi^{1}\right\},
\end{array}\right.
$$


and the right-hand sides of these equations can be regarded as lower order terms thank of (3.4), (3.14), (3.18), (3.20), and Lemma 3.2. Therefore, we can show the following lemma.

Lemma 4.1. Let $g, h, c_{0}, M_{0}$ be positive constants and $m$ an integer such that $m>\frac{n}{2}+1$. Suppose that the initial data $\left(\eta_{0}, \phi_{0}^{0}, \phi_{0}^{1}\right)$ and $b$ satisfy the conditions in (1.10), then for any $\varepsilon>0$ there exists a maximal existence time $T_{\varepsilon}>0$ such that the initial value problem (4.1)-(4.2) has a unique solution $\left(\eta^{\varepsilon}, \phi^{0, \varepsilon}, \phi^{1, \varepsilon}\right)$ satisfying

$$
\eta^{\varepsilon}, \nabla \phi^{0, \varepsilon} \in C\left(\left[0, T_{\varepsilon}\right) ; H^{m}\right), \quad \phi^{1, \varepsilon} \in C\left(\left[0, T_{\varepsilon}\right) ; H^{m+1}\right) .
$$

We are going to derive uniform estimates of the solution $\left(\eta^{\varepsilon}, \phi^{0, \varepsilon}, \phi^{1, \varepsilon}\right)$ with respect to the regularized parameter $\varepsilon>0$ for a time interval $[0, T]$ independent of $\varepsilon$. The following lemma gives a relation of the solution, which corresponds to the third equation in model system (1.1) (equivalently (3.2)). For simplicity of notation we write $\left(\eta, \phi^{0}, \phi^{1}\right)$ in place of $\left(\eta^{\varepsilon}, \phi^{0, \varepsilon}, \phi^{1, \varepsilon}\right)$ in the following. relation

Lemma 4.2. The solution $\left(\eta, \phi^{0}, \phi^{1}\right)$ obtained in Lemma 4.1 satisfies the

$$
\phi_{t}^{0}-\varepsilon \Delta \phi^{0}+H^{2}\left(\phi_{t}^{1}-\varepsilon \Delta \phi^{1}\right)+F_{1}=0
$$

where $F_{1}$ is defined by (3.4).

Proof. In view of the derivation of the first two equations in (3.21) from (3.10), we rewrite the first two equations in (4.1) as

$$
\left\{\begin{array}{l}
L_{0}\left(\phi_{t}^{0}-\varepsilon \Delta \phi^{0}\right)+\left(H^{-2} L_{22}-L_{12}\right)\left(H^{-2} F_{1}\right)=H^{-2} F_{2}, \\
L_{1}\left(\phi_{t}^{1}-\varepsilon \Delta \phi^{1}\right)+\left(H^{2} L_{11}-L_{12}^{*}\right) F_{1}=-F_{2} .
\end{array}\right.
$$

By the relation (3.9) of $L_{0}$ and $L_{1}$ the second equation in (4.4) is rewritten as

$$
L_{0}\left(H^{2}\left(\phi_{t}^{1}-\varepsilon \Delta \phi^{1}\right)\right)+\left(L_{11}-H^{-2} L_{12}^{*}\right) F_{1}=-H^{-2} F_{2} .
$$

Adding this and the first equation in (4.4) we obtain

$$
L_{0}\left(\phi_{t}^{0}-\varepsilon \Delta \phi^{0}+H^{2}\left(\phi_{t}^{1}-\varepsilon \Delta \phi^{1}\right)+F_{1}\right)=0 .
$$

Since the operator $L_{0}$ is invertible, the above equation gives the desired relation.

Concerning the generalized Rayleigh-Taylor sign condition, a regularized version of the function $a$ defined by (1.9) is given by

$$
\begin{aligned}
a^{\varepsilon}:= & g+2 H\left(\phi_{t}^{1}-\varepsilon \Delta \phi^{1}\right)+2 H^{3}\left|\nabla \phi^{1}\right|^{2}+2 H \nabla \phi^{0} \cdot \nabla \phi^{1}-2 \phi^{1} \nabla b \cdot \nabla \phi^{0} \\
& -6 H^{2} \phi^{1} \nabla b \cdot \nabla \phi^{1}+4 H\left(1+|\nabla b|^{2}\right)\left(\phi^{1}\right),
\end{aligned}
$$

where we have 


$$
\begin{aligned}
\phi_{t}^{1}-\varepsilon \Delta \phi^{1} & =-L_{1}^{-1}\left(F_{2}+\left(H^{2} L_{11}-L_{12}^{*}\right) F_{1}\right) \\
& =-L_{1}^{-1}\left(F_{2}-\frac{2}{3} H^{3} \Delta F_{1}+H^{2} \nabla b \cdot \nabla F_{1}\right),
\end{aligned}
$$

which comes from the second equation in (4.4) and (3.12). Therefore, in view of Remark 3.1 we see that $a^{\varepsilon}(x, 0)=a(x, 0)$ so that by the assumption (1.10) we have

$$
a^{\varepsilon}(x, 0) \geq c_{0} \quad \text { for } x \in \mathbf{R}^{n} .
$$

Moreover, by the definition (3.14) of the function $a_{1}$ and Lemma 4.2 it holds that

$$
\left\{\begin{array}{l}
a_{1}+2 H\left(\phi_{t}^{1}-\varepsilon \Delta \phi^{1}\right)=a^{\varepsilon}, \\
H a_{1}-2 F_{1}-2\left(\phi_{t}^{0}-\varepsilon \Delta \phi^{0}\right)=H a^{\varepsilon} .
\end{array}\right.
$$

Therefore, by the definition (3.17) of $P_{0}$ and $P_{1}$ and the explicit expressions (3.7) and (3.8) of $L_{0}$ and $L_{1}$, we rewrite (4.1) as

$$
\left\{\begin{array}{l}
-4 \nabla \cdot\left(H \nabla\left(\phi_{t}^{0}-\varepsilon \Delta \phi^{0}\right)\right)-4 \nabla \cdot\left(H(\boldsymbol{u} \cdot \nabla) \nabla \phi^{0}\right)+\nabla \cdot\left(H a^{\varepsilon} \nabla \eta\right)=\tilde{G}_{0} \\
-\frac{4}{5} \nabla \cdot\left(H^{5} \nabla\left(\phi_{t}^{1}-\varepsilon \Delta \phi^{1}\right)\right)-\frac{4}{5} \nabla \cdot\left(H^{5}(\boldsymbol{u} \cdot \nabla) \nabla \phi^{1}\right)-\nabla \cdot\left(H^{3} a^{\varepsilon} \nabla \eta\right)=\tilde{G}_{1} \\
9\left(\eta_{t}-\varepsilon \Delta \eta\right)+9 \boldsymbol{u} \cdot \nabla \eta-H \Delta \phi^{0}+H^{3} \Delta \phi^{1}=G_{2}
\end{array}\right.
$$

where $\tilde{G}_{0}$ and $\tilde{G}_{1}$ are collections of lower order terms, although they contain the time derivatives. More precisely,

$$
\left\{\begin{aligned}
\tilde{G}_{0}:= & G_{0}+2 \nabla \eta \cdot \nabla\left(\phi_{t}^{0}-\varepsilon \Delta \phi^{0}\right)+H^{-1}\left(10\left(1+|\nabla b|^{2}\right)+15 \nabla b \cdot \nabla H\right. \\
& \left.+6|\nabla H|^{2}-\frac{7}{4} H \Delta b\right)\left(\phi_{t}^{0}-\varepsilon \Delta \phi^{0}\right) \\
\tilde{G}_{1}:= & G_{1}+2 \nabla \eta \cdot \nabla\left(H^{4}\left(\phi_{t}^{1}-\varepsilon \Delta \phi^{1}\right)\right)+H^{3}\left(2\left(1+|\nabla b|^{2}\right)+3 \nabla b \cdot \nabla H\right. \\
& \left.-2|\nabla H|^{2}+\frac{5}{4} H \Delta b\right)\left(\phi_{t}^{1}-\varepsilon \Delta \phi^{1}\right) .
\end{aligned}\right.
$$

The following lemma gives a uniform estimate of the solution.

LEMma 4.3. There exist a time $T>0$ and a constant $C>0$ independent of $\varepsilon$ such that the solution $\left(\eta^{\varepsilon}, \phi^{\varepsilon}\right)$ obtained in Lemma 4.1 satisfies the uniform estimate

$$
\begin{aligned}
& \sup _{0 \leq t \leq T}\left(\left\|\eta^{\varepsilon}(t)\right\|_{m}^{2}+\left\|\nabla \phi^{0, \varepsilon}(t)\right\|_{m}^{2}+\left\|\phi^{1, \varepsilon}(t)\right\|_{m+1}^{2}\right) \\
& \quad+\varepsilon \int_{0}^{T}\left(\left\|\eta^{\varepsilon}(t)\right\|_{m+1}^{2}+\left\|\nabla \phi^{0, \varepsilon}(t)\right\|_{m+1}^{2}+\left\|\phi^{1, \varepsilon}(t)\right\|_{m+2}^{2}\right) \mathrm{d} t \leq C
\end{aligned}
$$

for $0<\varepsilon \leq 1$. 
Proof. Once again we simply write $\left(\eta, \phi^{0}, \phi^{1}\right)$ in place of $\left(\eta^{\varepsilon}, \phi^{0, \varepsilon}, \phi^{1, \varepsilon}\right)$. By (1.10), (3.4), (3.6), (3.12), (4.5), Lemma 3.2, and the Sobolev imbedding theorem, there exists a constant $C_{0}=C_{0}\left(g, h, c_{0}, M_{0}\right)>0$ such that

$$
c_{0} \leq H(x, 0) \leq C_{0}, \quad c_{0} \leq a^{\varepsilon}(x, 0) \leq C_{0} \quad \text { for } x \in \mathbf{R}^{n} .
$$

Now, we assume that

$$
\left\{\begin{array}{l}
\sup _{0 \leq t \leq T}\left(\|\eta(t)\|_{m}^{2}+\left\|\nabla \phi^{0}(t)\right\|_{m}^{2}+\left\|\phi^{1}(t)\right\|_{m+1}^{2}\right) \\
\quad+\varepsilon \int_{0}^{T}\left(\|\eta(t)\|_{m+1}^{2}+\left\|\nabla \phi^{0}(t)\right\|_{m+1}^{2}+\left\|\phi^{1}(t)\right\|_{m+2}^{2}\right) \mathrm{d} t \leq C, \\
c_{0} / 2 \leq H(x, t) \leq 2 C_{0}, \quad c_{0} / 2 \leq a^{\varepsilon}(x, t) \leq 2 C_{0} \quad \text { for } 0 \leq t \leq T, x \in \mathbf{R}^{n},
\end{array}\right.
$$

where the constant $C$ and the time $T$ will be defined later. Here and in what follows, positive constants depending on $g, h, m, c_{0}, M_{0}$ are denoted by the same symbol $C_{0}$ and positive constants depending also on $C$ are denoted by $C_{1}$.

Let $\alpha$ be a multi-index such that $1 \leq|\alpha| \leq m$. Applying the differential operator $\partial^{\alpha}=\left(\frac{\partial}{\partial x}\right)^{\alpha}$ to the equations in (4.9) and writing $\zeta^{\alpha}:=\partial^{\alpha} \eta, \psi^{0, \alpha}:=\partial^{\alpha} \phi^{0}$, and $\psi^{1, \alpha}:=\partial^{\alpha} \phi^{1}$, we obtain

$$
\left\{\begin{array}{l}
-4 \nabla \cdot\left(H \nabla \psi_{t}^{0, \alpha}\right)+4 \varepsilon \Delta\left(H \Delta \psi^{0, \alpha}\right)-4 \nabla \cdot\left(H(\boldsymbol{u} \cdot \nabla) \nabla \psi^{0, \alpha}\right)+\nabla \cdot\left(H a^{\varepsilon} \nabla \zeta^{\alpha}\right)=G_{0}^{\alpha}, \\
-\frac{4}{5} \nabla \cdot\left(H^{5} \nabla \psi_{t}^{1, \alpha}\right)+\frac{4}{5} \varepsilon \Delta\left(H^{5} \Delta \psi^{1, \alpha}\right)-\frac{4}{5} \nabla \cdot\left(H^{5}(\boldsymbol{u} \cdot \nabla) \nabla \psi^{1, \alpha}\right) \\
\quad-\nabla \cdot\left(H^{3} a^{\varepsilon} \nabla \zeta^{\alpha}\right)=G_{1}^{\alpha}, \\
9 a^{\varepsilon} \zeta_{t}^{\alpha}-9 \varepsilon \nabla \cdot\left(a^{\varepsilon} \nabla \zeta^{\alpha}\right)+9 a^{\varepsilon} \boldsymbol{u} \cdot \nabla \zeta^{\alpha}-\nabla \cdot\left(H a^{\varepsilon} \nabla \psi^{0, \alpha}\right)+\nabla \cdot\left(H^{3} a^{\varepsilon} \nabla \psi^{1, \alpha}\right)=G_{2}^{\alpha},
\end{array}\right.
$$

where

$$
\left\{\begin{aligned}
G_{0}^{\alpha}:= & \partial^{\alpha} \tilde{G}_{0}+\nabla \cdot\left\{4 \varepsilon(\nabla H) \Delta \partial^{\alpha} \phi^{0}+4\left[\partial^{\alpha}, H\right] \nabla\left(\phi_{t}^{0}-\varepsilon \Delta \phi^{0}\right)\right. \\
& \left.+4\left[\partial^{\alpha}, H(\boldsymbol{u} \cdot \nabla)\right] \nabla \phi^{0}-\left[\partial^{\alpha}, H a^{\varepsilon}\right] \nabla \eta\right\}, \\
G_{1}^{\alpha}:= & \partial^{\alpha} \tilde{G}_{1}+\nabla \cdot\left\{4 \varepsilon H^{4}(\nabla H) \Delta \partial^{\alpha} \phi^{1}+\frac{4}{5}\left[\partial^{\alpha}, H^{5}\right] \nabla\left(\phi_{t}^{1}-\varepsilon \Delta \phi^{1}\right)\right. \\
& \left.+\frac{4}{5}\left[\partial^{\alpha}, H^{5}(\boldsymbol{u} \cdot \nabla)\right] \nabla \phi^{1}-\left[\partial^{\alpha}, H^{3} a^{\varepsilon}\right] \nabla \eta\right\}, \\
G_{2}^{\alpha}:= & a^{\varepsilon}\left\{\partial^{\alpha} G_{2}-9\left[\partial^{\alpha}, \boldsymbol{u} \cdot \nabla\right] \eta+\left[\partial^{\alpha}, H\right] \Delta \phi^{0}-\left[\partial^{\alpha}, H^{3}\right] \Delta \phi^{1}\right\} \\
& -9 \varepsilon \nabla a^{\varepsilon} \cdot \nabla \partial^{\alpha} \eta-\nabla\left(H a^{\varepsilon}\right) \cdot \nabla \partial^{\alpha} \phi^{0}+\nabla\left(H^{3} a^{\varepsilon}\right) \cdot \nabla \partial^{\alpha} \phi^{1} .
\end{aligned}\right.
$$

The corresponding energy function $E^{\alpha}(t)$ and the dissipation function $F^{\alpha}(t)$ are defined by

$$
\left\{\begin{array}{l}
E^{\alpha}(t):=9\left(a^{\varepsilon} \zeta^{\alpha}, \zeta^{\alpha}\right)+4\left(H \nabla \psi^{0, \alpha}, \nabla \psi^{0, \alpha}\right)+\frac{4}{5}\left(H^{5} \nabla \psi^{1, \alpha}, \nabla \psi^{1, \alpha}\right), \\
F^{\alpha}(t):=9\left(a^{\varepsilon} \nabla \zeta^{\alpha}, \nabla \zeta^{\alpha}\right)+4\left(H \Delta \psi^{0, \alpha}, \Delta \psi^{0, \alpha}\right)+\frac{4}{5}\left(H^{5} \Delta \psi^{1, \alpha}, \Delta \psi^{1, \alpha}\right),
\end{array}\right.
$$


which are equivalent to $\left\|\zeta^{\alpha}(t)\right\|^{2}+\left\|\nabla \psi^{\alpha}(t)\right\|^{2}$ and $\left\|\nabla \zeta^{\alpha}(t)\right\|^{2}+\left\|\Delta \psi^{\alpha}(t)\right\|^{2}$, respectively, with $\psi^{\alpha}=\left(\psi^{0, \alpha}, \psi^{1, \alpha}\right)$. In fact, we easily see that

$$
\begin{aligned}
\frac{\mathrm{d}}{\mathrm{d} t} E^{\alpha}(t)+\varepsilon F^{\alpha}(t)= & \left(2 G_{2}^{\alpha}+9\left(a_{t}^{\varepsilon}+\nabla \cdot\left(a^{\varepsilon} \boldsymbol{u}\right)\right) \zeta^{\alpha}, \zeta^{\alpha}\right) \\
& +\left(2 G_{0}^{\alpha}, \psi^{0, \alpha}\right)+\left(4\left(H_{t}+\nabla \cdot(H \boldsymbol{u})\right) \nabla \psi^{0, \alpha}, \nabla \psi^{0, \alpha}\right) \\
& +\left(2 G_{1}^{\alpha}, \psi^{1, \alpha}\right)+\left(\left(4 H^{4} H_{t}+\frac{4}{5} \nabla \cdot\left(H^{5} \boldsymbol{u}\right)\right) \nabla \psi^{1, \alpha}, \nabla \psi^{1, \alpha}\right) \\
\leq & C_{0}\left(\left|a_{t}^{\varepsilon}\right|_{\infty}+\left|H_{t}\right|_{\infty}+\left|\nabla \cdot\left(a^{\varepsilon} \boldsymbol{u}\right)\right|_{\infty}+|\nabla \cdot(H \boldsymbol{u})|_{\infty}\right. \\
& \left.+\left|\nabla \cdot\left(H^{5} \boldsymbol{u}\right)\right|_{\infty}\right) E^{\alpha}(t)+2\left\|G_{2}^{\alpha}\right\|\left\|\zeta^{\alpha}\right\|+2\left\|\left(G_{0}^{\alpha}, G_{1}^{\alpha}\right)\right\|_{-1}\left\|\psi^{\alpha}\right\|_{1} .
\end{aligned}
$$

By (3.18) and (3.20) we have $\left\|\left(G_{0}, G_{1}\right)\right\|_{m-1}+\left\|G_{2}\right\|_{m} \leq C_{1}$, which together with (4.3) and Lemma 3.2 implies that $\left\|\phi_{t}-\varepsilon \Delta \phi\right\|_{m}+\left\|\eta_{t}-\varepsilon \Delta \eta\right\|_{m-1} \leq C_{1}$. Particularly, we get $\int_{0}^{T}\left(\left\|\phi_{t}\right\|_{m}^{2}+\left\|\eta_{t}\right\|_{m-1}^{2}\right) \mathrm{d} t \leq C_{1}(1+T)$, where $\phi=\left(\phi^{0}, \phi^{1}\right)$. It follows from (4.6) that

$$
\left(\phi_{t}^{1}-\varepsilon \Delta \phi^{1}\right)_{t}=\left(-L_{1}^{-1} \partial_{t}+L_{1}^{-1}\left[\partial_{t}, L_{1}\right] L_{1}^{-1}\right)\left(F_{2}-\frac{2}{3} H^{3} \Delta F_{1}+H^{2} \nabla b \cdot \nabla F_{1}\right),
$$

so that by Lemma 3.2 we obtain $\int_{0}^{T}\left\|\left(\phi_{t}^{1}-\varepsilon \Delta \phi^{1}\right)_{t}\right\|_{m-1}^{2} \mathrm{~d} t \leq C_{1}$. Therefore, by (4.5) we obtain $\int_{0}^{T}\left\|a_{t}^{\varepsilon}\right\|_{m-1}^{2} \mathrm{~d} t \leq C_{1}$. By the Sobolev imbedding theorem we get $\frac{\mathrm{d}}{\mathrm{d} t} E^{\alpha}(t)+\frac{\varepsilon}{2} F^{\alpha}(t) \leq C_{1}\left(1+\left\|a_{t}^{\varepsilon}(t)\right\|_{m-1}+\left\|\eta_{t}(t)\right\|_{m-1}\right)$. Integrating this with respect to $t$ yields that $E^{\alpha}(t)+\frac{\varepsilon}{2} \int_{0}^{t} F^{\alpha}(\tau) \mathrm{d} \tau \leq C_{0}+C_{1}(t+\sqrt{t})$. For the lowest order terms, it is easy to see that $\|\eta\|_{m-1}+\left\|\nabla \phi^{0}\right\|_{m-1}+\left\|\phi^{1}\right\|_{m} \leq C_{0}+C_{1}(t+\sqrt{t})$. To summarize, we have obtained the estimates

$$
\left\{\begin{array}{l}
\|\eta(t)\|_{m}^{2}+\left\|\nabla \phi^{0}(t)\right\|_{m}^{2}+\left\|\phi^{1}(t)\right\|_{m+1}^{2} \\
\quad+\varepsilon \int_{0}^{t}\left(\|\eta(\tau)\|_{m+1}^{2}+\left\|\nabla \phi^{0}(\tau)\right\|_{m+1}^{2}+\left\|\phi^{1}(\tau)\right\|_{m+2}^{2}\right) \mathrm{d} \tau \leq C_{0}+C_{1}(t+\sqrt{t}), \\
|H(x, t)-H(x, 0)|+\left|a^{\varepsilon}(x, t)-a^{\varepsilon}(x, 0)\right| \leq C_{1}(t+\sqrt{t}) \text { for } x \in \mathbf{R}^{n} .
\end{array}\right.
$$

Now, we define the constant $C$ by $C=2 C_{0}$, and then the time $T$ sufficiently small so that $C_{1}(T+\sqrt{T}) \ll 1$. Then, we see that (4.13) holds. The proof is complete.

Once we obtain this kind of uniform estimates, we can pass to the limit $\varepsilon \rightarrow+0$ in the regularized problem (4.1)-(4.2) and obtain the following lemma.

LEMMA 4.4. Let $g, h, c_{0}, M_{0}$ be positive constants and $m$ an integer such that $m>\frac{n}{2}+1$. There exists a time $T>0$ such that if the initial data $\left(\eta_{0}, \phi_{0}^{0}, \phi_{0}^{1}\right)$ and 
$b$ satisfy the conditions in (1.10), then the initial value problem (3.21)-(3.22) has a unique solution $\left(\eta, \phi^{0}, \phi^{1}\right)$ satisfying

$$
\eta, \nabla \phi^{0} \in C\left([0, T] ; H^{m}\right), \quad \phi^{1} \in C\left([0, T] ; H^{m+1}\right) .
$$

\section{Proof of the main theorem}

Now, we are going to show that the solution to the transformed problem (3.21)-(3.22) is the solution of the model system (1.1) and (1.3) if the initial data satisfy the necessary condition (1.6). Let $\left(\eta, \phi^{0}, \phi^{1}\right)$ be the solution of (3.21)(3.22) obtained in Lemma 4.4 and define functions $R_{1}, R_{2}, R_{3}$ by the left-hand sides of the equations in (1.1) (equivalently (3.2)), that is,

$$
\left\{\begin{array}{l}
R_{1}:=\eta_{t}-L_{11} \phi^{0}-L_{12} \phi^{1} \\
R_{2}:=H^{2} \eta_{t}-L_{12}^{*} \phi^{0}-L_{22} \phi^{1} \\
R_{3}:=\phi_{t}^{0}+H^{2} \phi_{t}^{1}+F_{1}
\end{array}\right.
$$

It is sufficient to show that $\left(R_{1}, R_{2}, R_{3}\right) \equiv \mathbf{0}$. We also define a function $R_{4}$ by

$$
R_{4}:=H^{2}\left(L_{11} \phi^{0}+L_{12} \phi^{1}\right)-\left(L_{12}^{*} \phi^{0}+L_{22} \phi^{1}\right) .
$$

Then, the necessary condition (1.6) is equivalent to $R_{4} \equiv 0$. It is easy to see that

$$
R_{4}=-H^{2} R_{1}+R_{2}
$$

We will drive partial differential equations for $R_{1}, R_{2}, R_{3}, R_{4}$ by using the fact that $\left(\eta, \phi^{0}, \phi^{1}\right)$ is the solution of the transformed equations (3.21). To this end, we follow essentially the calculation in the derivation of (3.21). Differentiating (5.2) with respect to $t$ and using the first equation in (5.1) to eliminate $\eta_{t}$ we obtain

$$
\left(H^{2} L_{11}-L_{12}^{*}\right) \phi_{t}^{0}-\left(L_{22}-H^{2} L_{12}\right) \phi_{t}^{1}=F_{2}+R_{4 t}+F_{3} R_{1},
$$

which corresponds to (3.5). It follows from the third equation in (5.1) that $\phi_{t}^{1}=$ $-H^{-2}\left(\phi_{t}^{0}+F_{1}-R_{3}\right)$ and $\phi_{t}^{0}=-H^{2} \phi_{t}^{1}-F_{1}+R_{3}$. Plugging these into (5.4) we obtain

$$
\left\{\begin{array}{c}
L_{0} \phi_{t}^{0}+\left(H^{-2} L_{22}-L_{12}\right)\left(H^{-2} F_{1}\right) \\
\quad=H^{-2} F_{2}+\left(H^{-2} L_{22}-L_{12}\right)\left(H^{-2} R_{3}\right)+H^{-2}\left(R_{4 t}+F_{3} R_{1}\right) \\
L_{1} \phi_{t}^{1}+\left(H^{2} L_{11}-L_{12}^{*}\right) F_{1}=-F_{2}+\left(H^{2} L_{11}-L_{12}^{*}\right) R_{3}-\left(R_{4 t}+F_{3} R_{1}\right)
\end{array}\right.
$$

which corresponds to (3.10). It follows from the first equation in (5.1) and (5.2) that

$$
9 \eta_{t}+9 \boldsymbol{u} \cdot \nabla \eta-H \Delta \phi^{0}+H^{3} \Delta \phi^{1}=G_{2}+9 R_{1}+15 H^{-2} R_{4}
$$


which corresponds to (3.19). By using the fact that $\left(\eta, \phi^{0}, \phi^{1}\right)$ is the solution of the transformed equations (3.21) and the identities in (5.5) and (5.6), we obtain

$$
\left\{\begin{array}{l}
\left(H^{-2} L_{22}-L_{12}\right)\left(H^{-2} R_{3}\right)+H^{-2}\left(R_{4 t}+F_{3} R_{1}\right)=0 \\
\left(H^{2} L_{11}-L_{12}^{*}\right) R_{3}-\left(R_{4 t}+F_{3} R_{1}\right)=0 \\
9 R_{1}+15 H^{-2} R_{4}=0
\end{array}\right.
$$

It follows from the first two equations that $L_{0} R_{3}=0$, which implies that $R_{3}=0$ due to the invertibility of $L_{0}$. Thus, by the first two equations again, we obtain $R_{4 t}+F_{3} R_{1}=0$, which together with the third equation yields that

$$
\frac{\mathrm{d}}{\mathrm{d} t} R_{4}=\frac{5}{3} H^{-2} F_{3} R_{4}
$$

that is, $R_{4}$ satisfies this linear homogeneous ordinary differential equation in $t$. Therefore, by the uniqueness of the solution to this simple equation we see that $\left.R_{4}\right|_{t=0}=0$ implies $R_{4}=0$ for all $t$. This means that the manifold defined by the relation (1.6) in a phase space of $\left(\eta, \phi^{0}, \phi^{1}\right)$ is invariant under the time evolution by the transformed equations (3.21). Now, by the third equation in (5.7) and (5.3) we obtain $R_{1}=R_{2}=0$. Therefore, we have shown that $\left(\eta, \phi^{0}, \phi^{1}\right)$ is the solution of the model system (1.1) and (1.3).

Finally, we will show the conservation of the energy.

LEMmA 5.1. Let $\left(\eta, \phi^{0}, \phi^{1}\right)$ be a smooth solution of the model system (1.1) (equivalently (3.2)). Then, the energy function $E(t)$ defined by (1.7) is a conserved quantity.

Proof. In view of the definition (1.7) and the identity (3.23) we see that

$$
\frac{\mathrm{d}}{\mathrm{d} t} E(t)=\left(L_{11} \phi^{0}+L_{12} \phi^{1}, \phi_{t}^{0}\right)+\left(L_{12}^{*} \phi^{0}+L_{22} \phi^{1}, \phi_{t}^{1}\right)+\left(F_{1}, \eta_{t}\right)=0,
$$

where we used that fact that $L_{11}$ and $L_{22}$ are symmetric in $L^{2}\left(\mathbf{R}^{n}\right)$.

Acknowledgement. The second author would like to thank Professor Taro Kakinuma at Kagoshima University for fruitful discussions. His questions concerning the model system analyzed in the present paper were the starting point for the investigations presented here.

\section{REFERENCES}

[1] G. B. AIRY, Tides and waves, Encyclopaedia metropolitana 5, London, 1845, 241-396.

[2] J. T. Beale, T. Y. Hou and J. S. Lowengrub, Growth rates for the linearized motion of fluid interfaces away from equilibrium, Commun. Pure Appl. Math. 46 (1993), 12691301 . 
[ 3 ] T. B. Benjamin, Internal waves of permanent form in fluids of great depth, J. Fluid Mech. 29 (1967), 559-592.

[ 4 ] T. B. Benjamin, J. L. Bona And J. J. Mahony, Model equations for long waves in nonlinear dispersive systems, Philos. Trans. Roy. Soc. London Ser. A. 272 (1972), 47-78.

[5] J. BoussinesQ, Théorie des ondes et des remous qui se propagent le long d'un canal rectangulaire horizontal, en communiquant au liquide contenu dans ce canal des vitesses sensiblement pareilles de la surface au fond, J. Math. Pure. Appl. 17 (1872), 55-108.

[6] R. Camassa and D. D. Holm, An integrable shallow water equation with peaked solitons, Phys. Rev. Lett. 71 (1993), 1661-1664.

[7] D. Clamond AND D. DutyKh, Practical use of variational principles for modeling water waves, Phys. D 241 (2012), 25-36.

[8] K. O. Friedrichs, On the derivation of the shallow water theory, Appendix to: "The formulation of breakers and bores" by J. J. Stoker in Comm. Pure Appl. Math. 1 (1948), $1-87$.

[9] B. FuChSSTEINER AND A. S. FoKAS, Symplectic structures, their Bäcklund transformations and hereditary symmetries, Phys. D 4 (1981/82), 47-66.

[10] A. E. Green, N. Laws and P. M. Naghdi, On the theory of water waves, Proc. Roy. Soc. Lond. Ser. A. 338 (1974), 43-55.

[11] A. E. Green And P. M. NAGHDI, Derivation of equations for wave propagation in water of variable depth, J. Fluid Mech. 78 (1976), 237-246.

[12] M. Isobe, A proposal on a nonlinear gentle slope wave equation, Proceedings of Coastal Engineering, JSCE 41 (1994), 1-5 (in Japanese).

[13] M. Isobe, Time-dependent mild-slope equations for random waves, Proceedings of 24th International Conference on Coastal Engineering, ASCE, 1994, 285-299.

[14] B. B. Kadomtsev and V. I. Petviashvili, On the stability of solitary waves in weakly dispersive media, Sov. Phys. Dokl. 15 (1970), 539-541.

[15] T. Kakinuma, Extension to internal waves in nonlinear gentle slope equation, Proceedings of Coastal Engineering, JSCE 47 (2000), 1-5 (in Japanese).

[16] T. KAKINUmA, A set of fully nonlinear equations for surface and internal gravity waves, Coastal engineering V: Computer modelling of seas and coastal regions, WIT Press, 2001, 225-234.

[17] T. KaKinuma, A nonlinear numerical model for surface and internal waves shoaling on a permeable beach, Coastal engineering VI: Computer modelling and experimental measurements of seas and coastal regions, WIT Press, 2003, 227-236.

[18] D. J. Korteweg AND G. DE VRIES, On the change of form of long waves advancing in a rectangular canal, and on a new type of long stationary waves, Phil. Mag. Ser. 5. 39 (1895), $422-443$.

[19] D. Lannes, Well-posedness of the water-waves equations, J. Amer. Math. Soc. 18 (2005), 605-654.

[20] J. C. Luke, A variational principle for a fluid with a free surface, J. Fluid Mech. 27 (1967), 395-397.

[21] H. ONO, Algebraic solitary waves in stratified fluids, J. Phys. Soc. Japan 39 (1975), 10821091.

[22] D. H. Peregrine, Calculations of the development of an undular bore, J. Fluid Mech. 25 (1966), 321-330.

[23] S. Wu, Well-posedness in Sobolev spaces of the full water wave problem in 2-D, Invent. Math. 130 (1997), 39-72.

[24] S. Wu, Well-posedness in Sobolev spaces of the full water wave problem in 3-D, J. Amer. Math. Soc. 12 (1999), 445-495. 
Yuuta Murakami

FORMER ADDRESS:

DePARTMENT OF MATHEMATICS

Faculty of Science and Technology, KeIo University

3-14-1 Hiyoshi, KoHOKU-KU, Yоконама, 223-8522

JAPAN

E-mail: murakami@johoku.ac.jp

Tatsuo Iguchi

Department of Mathematics

Faculty of Science and Technology, Keio University

3-14-1 HiYoshi, KoHOKU-KU, YокоHAMA, 223-8522

JAPAN

E-mail: iguchi@math.keio.ac.jp 